\title{
Clinico-Pathological Profile of Cutaneous Lupus Erythematosus Patients: A Report from A Tertiary Care Center of Chattogram
}

Mohammad Rafiqul Mowla ${ }^{1 *}$

Deva Pratim Barua'

Shakila Zaman ${ }^{1}$

Mohammad Ismail Hossain Chowdhury ${ }^{1}$ Papri Dutta ${ }^{2}$

Md. Wazed Ali Mridha ${ }^{3}$

'Department of Dermatology and Venereology Chittagong Medical College Chattogram, Bangladesh.

${ }^{2}$ Department of Dermatology and Venereology Southern Medical College Chattogram, Bangladesh.

${ }^{3}$ Department of Dermatology and Venereology Khulna Medical College Khulna, Bangladesh.
*Correspondence to:

Dr. Mohammad Rafiqul Mowla Associate Professor

Department of Dermatology and Venereology Chittagong Medical College

Chattogram. Bangladesh.

Mobile : +8801711341405

Email : rafiqulmowla66@yahoo.com

Date of Submission : 05.06 .2021

Date of Acceptance : 27.06 .2021

\begin{abstract}
Background : Nearly all epidemiologic studies have involved patients with Systemic Lupus Erythematosus (SLE). Few authors have investigated the characteristics of patients with Cutaneous Lupus Erythematosus (CLE). We aim to describe the epidemiologic, clinical, and immunologic characteristics of a series of patients diagnosed with CLE.
\end{abstract}

Materials and methods : This is a descriptive retrospective cross-sectional study carried out using the register records of total 218 patients attending the 'Lupus Clinic' in Chittagong Medical College Hospital during the period 2010 and 2020. The disease activity and damage of CLE were assessed according to the Cutaneous Lupus Erythematosus Disease Area and Severity Index (CLASI).

Results : There were 187 females (86\%) and 31 males (14\%) with the female: male ratio being 6.1:1. The mean age was $28 \pm 10.06$ (mean $\pm S D$ ) ranging between 11 and 65 years. The Chronic Cutaneous Lupus Erythematosus (CCLE) patients accounted 154 (71\%) followed by Acute Cutaneous Lupus Erythematosus (ACLE) 46 (21\%) and Subacute Cutaneous Lupus Erythematosus (SCLE) 18 (8\%). On the basis of CLASI score, 91 (42\%) patients were in mild form, 85 (39\%) in moderate form and 42 (19\%) in severe state. In LE specific skin lesions, common manifestation was photosensitivity 198 (91\%) followed by discoid rash 154 (71\%) and maculo-papular lupus rash 55 (25\%). Oral ulcer was seen in 49 (22\%) patients and malar rash in 46 (21\%) patients. Other observed LE specific skin manifestations were papulo squamous rash 11 (5\%), Toxic epidermal necrolysis like lesions 7 (3\%) and lichenoid lesions $6(3 \%)$. Among LE nonspecific skin lesions, common manifestation was nonscarring alopecia 123 (56\%) followed by Raynaud's phenomenon 17 (8\%) livedo reticularis 17 (8\%) Vasculitis 15 (7\%) Periungual telangiectasia 7 (3\%) erythema multiforme 6 (3\%) and leg Ulcer 5 (2\%). Antinuclear Antibody (ANA) 132 (61\%) was the most common autoantibody followed by anti-ds DNA 91 (42\%) anti-Sm antibody 2 (1\%) anti-phospholipid antibodies 9 (4\%) and anti-RNP 3 (1\%). Hematological manifestations were seen in 161 (73.85\%) where erythrocyte sedimentation rate was the most common hematological abnormality 161(73.85\%) followed by lymphopenia 126 (57.80\%) leucopenia 113 (51.80\%) thrombocytopenia 107 (49.10\%) anemia 92 (42\%) monocytopenia 37 (16.97\%).

Conclusions: CCLE was the most common subtypes of CLE. Patients with different subtypes of CLE have distinct clinical and pathological characteristics. The onset or concurrence of SLE mandates the involvement of other disciplines depending on organ involvement. In the absence of consensus on a definition that makes it possible to differentiate cutaneous forms of LE from SLE, the dermatologist's role in the correct diagnosis and classification of such patients is fundamental.

Key words: Cutaneous Lupus Erythematosus (CLE); Dermatologic manifestation; Skin lesion; Systemic Lupus Erythematosus (SLE). 


\section{INTRODUCTION}

Cutaneous Lupus Erythematosus (CLE) is a chronic, relapsing autoimmune condition encompassing a wide range of dermatologic manifestations. Skin involvement in CLE patients can be divided into two categories based on histology: Lupus Erythematosus- (LE-) specific and LE-nonspecific skin lesions. The presence of LE-specific lesions is necessary to confirm the diagnosis of CLE. LE-specific skin lesions are divided into several subtypes based on clinical characteristics: Acute CLE (ACLE) Subacute CLE (SCLE) and Chronic CLE (CCLE) with several variants including Discoid LE (DLE) presenting as a localized or generalized form, LE Profundus (LEP) (Also called lupus panniculitis or subcutaneous LE) Hypertrophic LE (HLE) Chilblain LE (CHLE) and Lupus Erythematosus Tumidus (LET) $)^{1,2}$.

Lupus Erythematosus (LE) is a complex autoimmune disease with a worldwide distribution and an unknown etiology ${ }^{3}$. It is characterized by a great clinical polymorphism and female predominance $e^{4,5}$. The appearance, progression and outcome of LE are influenced by genetic, immunological and environmental factors ${ }^{6}$. Ethnicity also seems to contribute to the expression and heterogeneity of the clinical and immunological features of disease ${ }^{7}$.

However, few studies have investigated the characteristics of patients with CLE. Most studies of patients with LE have focused on patients with Systemic Lupus erythematosus (SLE) and very few studies have been carried out on the various subtypes of $\mathrm{CLE}^{8-12}$. Epidemiologic data are fundamental to our understanding of the risk and burden of disease in the population. However, such data are challenging and resource intensive to derive in a fragmented health care system and for diseases such as LE, in which a heterogeneous constellation of clinical and laboratory features is necessary to establish a diagnosis. Inadequate data on clinico-pathological manifestations pose a major barrier to further understand this disease. Most of the data available are from the western population. Furthermore, the immune status, individual response to disease and type of antibodies, vary from person to person, place to place and population to population. In this study, we aim to describe the clinical and pathological characteristics of a series of patients diagnosed with CLE who were treated in a specialized unit of a tertiary care teaching hospital of Chattogram, Bangladesh.

\section{MATERIALS AND METHODS}

The present investigation is a descriptive retrospective crosssectional study carried out using the register records of patients attending the 'Lupus Clinic' in Chittagong Medical College Hospital $(\mathrm{CMCH})$ during the period 2010 and 2020. $\mathrm{CMCH}$ is the oldest tertiary care teaching hospital of the country. The 'Lupus Clinic' of $\mathrm{CMCH}$ caters for the patients from the Chittagong city as well as from neighboring districts and a multi-disciplinary specialized team is available at the 'Lupus
Clinic' of CMCH. The patients' cards were studied and the following clinical data were recorded: demographic characteristics, extent of skin involvement and serological findings were collected. Socio-demographic data included age, sex, completed education, living place (Rural/urban) monthly family income and smoking status.

A total number of 218 inpatients and outpatients with cutaneous involvement during the course of LE were included in the study. Data were obtained by questionnaires filled in by patients during their routine visits to the 'Lupus clinic' and by extracting medical records. Diagnosis of CLE has been established based on clinical manifestation and skin biopsy, if necessary. Patients were classified into 3 CLE subtypes: Acute Cutaneous LE (ACLE) Subacute Cutaneous LE (SCLE) or Chronic Cutaneous LE (CCLE) according to Sontheimer and Kuhn et $\mathrm{al}^{2,13}$. The disease activity and damage of CLE were assessed according to the Cutaneous Lupus Erythematosus Disease Area and Severity Index (CLASI) ${ }^{14}$.

The study protocol excluded patients who met the criteria for SLE but did not have LE-specific cutaneous manifestations, and patients who had clinical findings consistent with CLE but whose diagnosis was not confirmed on follow-up.

Routine blood, urinalysis, and other biochemical tests were performed. Chest X-ray and echocardiography and electrocardiography were performed in the recommended patients. C3 and C4 levels were measured if needed. Tests for Antinuclear Antibody (ANA) and anti-ds DNA antibodies were performed by Indirect Immunofluorescence Assay (IFA). Autoantibodies to Extractable Nuclear Antigens (ENA) (Sm and $\mathrm{Sm} / \mathrm{RNP}$ ) were studied where applicable by EnzymeLinked Immunosorbent Assay (ELISA). The IFA and ELISA tests were performed on an automated system.

After collection data were entered into Microsoft Excel spread sheet to generate a master sheet. Then they were fed into Statistical Package for Social Science (SPSS) version 23 for processing and analysis. Descriptive statistics included frequencies, mean, standard deviation, median, minimal, and maximal values. Continuous data were reported as the means \pm Standard Deviations (SD) and with regard to categorical ones, we used number and percentages. Proportions were presented with $95 \%$ Confidence Intervals ( $95 \%$ CI).

The authors certify that they have obtained all appropriate patient consent forms both in oral and written. The study was conducted in accordance with the Data Protection Act and according to the ethical guidelines of the Declaration of Helsinki and was approved by the Ethics Committee at Chittagong Medical College, Bangladesh.

\section{RESULTS}

There were 187 females (86\%) and 31 males (14\%) with the female: male ratio being 6.1:1. The mean age was $28 \pm 10.06$ (Mean $\pm \mathrm{SD}$ ) ranging between 11 and 65 years. Majority of the patients $142(65 \%)$ were from rural area and $76(35 \%)$ from urban area. Most of the male patients 29 (94\%) were smoker (Table I). 
Table I : Demographic characteristics of the patients $(\mathrm{n}=218)$

$\begin{array}{llr}\text { Characteristics } & & \text { Frequency }(\%, 95 \% \mathrm{CI}) \\ \text { Age } & \leq 18 \text { years } & 32(14.7 \%, 10.3-20.1 \%) \\ & 18-50 \text { years } & 170(78.0 \%, 71.9-83.3 \%) \\ & >50 \text { years } & 16(7.3 \%, 4.3-11.7 \%) \\ \text { Sex } & \text { Male } & 31(14.2 \%, 9.9-19.6 \%) \\ & \text { Female } & 187(85.8 \%, 80.4-90.1 \%) \\ \text { Educational status } & \text { Primary } & 53(24.3 \%, 18.8-30.6 \%) \\ & \text { Secondary } & 121(55.5 \%, 48.6-62.2 \%) \\ & \text { Above Secondary } & 44(20.2 \%, 15.1-26.1 \%) \\ \text { Smoker } & \text { Male }(\mathrm{n}=31) & 29(93.5 \%, 78.6-99.2 \%) \\ & \text { Female (n=187) } & 1(0.01 \%, 0.0-2.9 \%) \\ \text { Place of residence } & \text { Rural } & 142(65.1 \%, 58.4-71.5 \%) \\ & \text { Urban } & 76(34.9 \%, 28.6-41.6 \%) \\ \text { MFI (USD) } & <100 & 94(43.1 \%, 36.6-50.0 \%) \\ & 100-200 & 65(29.8 \%, 23.8-36.4 \%) \\ & >200 & 59(27.1 \%, 21.3-33.5 \%)\end{array}$

MFI: Monthly Family Income, USD: U S Dollar

CI: Confidence Interval.

Of the 218 patients with CLE, the CCLE patients counted 154 (71\%) which was higher than other forms of CLE followed by ACLE 46 (21\%) and SCLE 18 (8\%).

In LE specific skin lesions, common manifestation was photosensitivity 198 (91\%), followed by discoid rash 154 (71\%) and maculo-papular lupus rash 55 (25\%). Oral ulcer was seen in $49(22 \%)$ patients and malar rash in $46(21 \%)$ patients. Other observed LE specific skin manifestations were papulo squamous rash $11(5 \%)$, Toxic Epidermal Necrolysis (TEN) like lesions 7 (3\%) lichenoid lesions 6 (3\%). Among LE nonspecific skin lesions, common manifestation was nonscarring alopecia 123 (56\%) followed by Raynauds phenomenon 17 (8\%) livedo reticularis 17 (8\%) Vasculitis 15 (7\%) Periungual telangiectasia $7(3 \%)$ erythema multiforme 6 (3\%) leg Ulcer 5 (2\%) (Table II). Urticaria, panniculitis, acanthosis nigricans, rheumatoid nodules, anetoderma and lichen planus were less common.

Table II : Clinical characteristics of skin lesions (Both LE specific and Nonspecific)

$\begin{array}{lrrr}\text { CLE sub types } & \text { Frequency }(\%),(\mathrm{Cl}) & \text { Male }(\%),(\mathrm{Cl}) & \text { Female }(\%),(\mathrm{CI}) \\ \text { ACLE } & 46(21 \%) & 1(0.46 \%) & 45(24.1 \%) \\ & (15.9-27.1 \%) & (0.0-16.7 \%) & (18.1-30.8 \%) \\ \text { SCLE } & 18(8 \%) & 2(0.92 \%) & 16(7.34 \%) \\ & (4.9-12.7 \%) & (0.1-21.4 \%) & (5.0-13.5 \%) \\ \text { CCLE } & 154(71 \%) & 28(12.85 \%) & 126(57.8 \%) \\ & (64.1-76.6 \%) & (74.2-98.0 \%) & (60.2-74.0 \%)\end{array}$

LE specific skin lesions

Photosensitivity

Discoid Rash

Maculo-papular lupus rash

Oral ulcer

Malar rash
Frequency (\%) $198(90.8 \%)$

$154(71 \%)$

$55(25.2 \%)$

$49(22.5 \%)$

$46(21.1 \%)$

Papulo squamous rash

TEN like lesions

Lichenoid lesions

LE Nonspecific skin lesions

Non-scaring Alopecia

Livedo reticularis

Raynaud's phenomenon

Vasculitis

Periungual telangiectasia

Erythema Multiforme

Leg Ulcer

\# $\mathrm{n}$ will not correspond to $100 \%$, because of multiple cutaneous lesions.

*Figure in the parenthesis indicate corresponding percentage $\%$.

Of the total of 218 patients studied, 132 (61\%) patients had positive ANA. Anti-ds DNA antibodies were seen in 91 (42\%) patients. Anti-Sm antibodies were found in $2(1 \%)$ patients. Antiphospholipid antibodies were positive in $9(4 \%)$ and anti-RNP Ab in $3(1 \%)$ patients. Complement $3(\mathrm{C} 3)$ and complement 4 (C4) were decreased in 5 ( $2 \%$ ) patients (Table III).

Hematological manifestations were seen in 161 (73.85\%) patients. Increased Erythrocyte Sedimentation Rate (ESR) was the most common hematological abnormality 161(73.85\%) followed by lymphopenia 126 (57.80\%), leucopenia 113 (51.80\%), thrombocytopenia $107(49.10 \%)$ anemia 92 (42\%) and monocytopenia 37 (16.97\%). lymphocytosis occurred in 12 (5.51\%) patients (Table III).

A total of 49 (22\%) patients presented with albuminuria. Red Blood Cell (RBC) was found in the urine of 37 (17\%) patients. More than 0.5 gram proteins in 24 hours urine sample (24 hours UTP) were positive in 36 (17\%) patients (Table III).

In all, 218 patients 61 (28\%) had meet $\geq 4$ ACR criteria for SLE. Articular manifestations occurred in $54(25 \%)$ patients. Renal manifestations were presented in $36(17 \%)$ patients. Respiratory involvement was recorded in $28(13 \%)$ patients. Twelve $(6 \%)$ patients had neurological features (Figure- 1 and Table III).

Table III : Pathological profile of patients stratified by $\operatorname{sex}(n=218)$

Variables Total $(\mathrm{n}=218) \quad$ Male $(\mathrm{n}=31) \quad$ Sex $\quad$ Female $(\mathrm{n}=187)$

Anemia

Leucopenia

Thrombocytopenia

Lymphopenia

Raised ESR

Antinuclear antibodies

Anti-dsDNA antibodies

Proteinuria

Hematuria

$24 \mathrm{hUTP}>0.5 \mathrm{~g} / \mathrm{d}$

Patients fulfilling SLICC-2012

criteria for SLE

Data were expressed as frequency (Percentage) with $95 \%$ confidence interval of the proportion

Abbreviations: ESR: Erythrocyte sedimentation rate. UTP : Urinary Total Protein. 


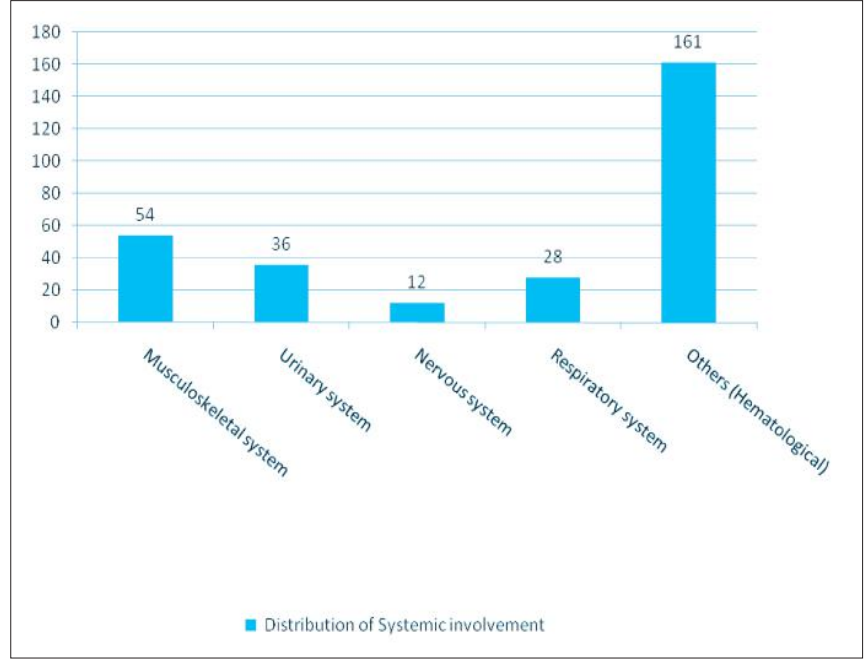

Figure 1 : Distribution of systemic involvement $(\mathrm{n}=218)$

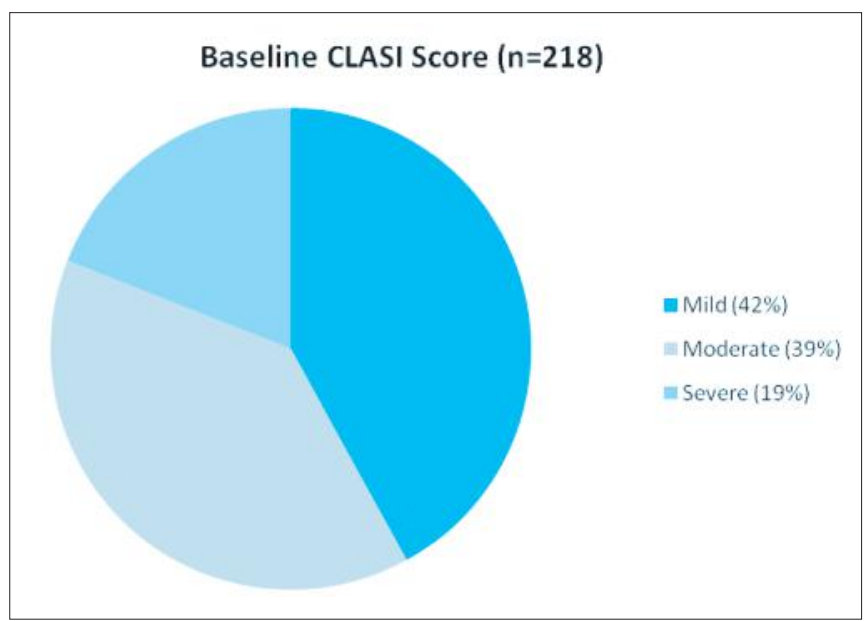

Figure 2 : Disease severity in Cutaneous Lupus Erythematosus patients (CLASI score)

On the basis of CLASI score, 91 (42\%) patients were in mild form, $85(39 \%)$ in moderate form and $42(19 \%)$ in severe state (Figure- 2 ).

\section{DISCUSSION}

Among the patients female 187 (86\%) male 31 (14\%) with a sex ratio of $6.1: 1$. The mean age of onset of disease was 28 years (Range 11-65 years). These findings are similar to Indian studies by Kishor $\mathrm{N}$ et al and Binoy JP et al where they conducted study on SLE patients ${ }^{15,16}$. Other clinical studies have consistently demonstrated a female predominance also. In general, this percentage ranges from $78 \%$ to $96 \%$ in most studies, with a female-male ratio of approximately 10:17, 18 . This excess of females is especially noteworthy in the 15- to 64-year-old age group, where ratios of age- and sex-specific incidence rates show a 6- to 10 -fold female excess. This age related differences in the female-male ratios have been considered to be related to hormonal changes ${ }^{19}$. LE can appear in people of any age. It is interesting to note that, in several studies, patient age at the beginning of symptoms can modify the clinical and pathological characteristics of LE.
In LE specific skin lesions, common manifestation was photosensitivity 198 (91\%), followed by discoid rash 154 (71\%) and maculo-papular lupus rash 55 (25\%). The prevalence of photosensitivity ranges from $28 \%$ to $71 \%{ }^{19}$. It is one of the major diagnostic criteria for SLE. Photosensitivity precedes the clinical onset of internal manifestations of SLE in about one third of patients. Some patients may not notice erythema after prolonged UV exposure, but hours or days later they may note increased arthralgia, malaise, or fever. CLE is frequently a photosensitive eruption that can be induced by both Ultraviolet A (UVA) and Ultraviolet B (UVB) light. In patients with DLE, SCLE and SLE, the action spectrum of induced lesions was in UVB range in 33\%, in UVA range in $14 \%$ and in both UVA and UVB in 53\%. CLE are photosensitive, therefore, disease prevalence might be higher in areas with more ambient sun exposure. It is important to use both full sleeves clothes and broad spectrum sunscreen and avoidance of both natural and artificial UV light exposures. There is often a latency period of several weeks between UV exposure and disease symptoms so it is important to repeatedly inform the patients about this association ${ }^{20}$.

Among LE nonspecific skin lesions, common manifestation was non-scarring alopecia $123(56 \%)$ followed by Raynauds phenomenon 17 (8\%) livedo reticularis 17 (8\%) Vasculitis 15 (7\%) Periungual telangiectasia 7 (3\%) erythema multiforme 6 (3\%) leg Ulcer $5(2 \%)$ urticarial $2(1 \%)$ and acanthosis nigricans 2 (1\%). On the basis of CLASI score, 91 (42\%) patients were in mild form, $85(39 \%)$ in moderate form and 42 $(19 \%)$ in severe state (Figure- 2). Hair loss is a common and characteristic finding in patients with LE. It may be scarring, if preceded by DLE or nonscarring. Urticaria, angioedema, and Raynaud's phenomenon are common cutaneous vascular reaction patterns. Some patients with LE described lesions suggestive of urticarial vasculitis, with prevalences ranging from $7 \%$ to $22 \%{ }^{21,22}$. Dermal vasculitis has been reported in $18 \%$ to $70 \%$ of patients with LE. Livedo reticularis may be associated with the antiphospholipd syndrome and has been reported as an initial manifestation of LE in many patients ${ }^{23}$. Other skin disorders have been occasionally reported in patients with LE, such as erythema multiforme, chronic ulcers, splinter hemorrhages, rheumatoid nodules, and acanthosis nigricans. Mucous membrane involvement was thought to be relatively uncommon in LE. Dubois and Tuffanelli found only a $9 \%$ incidence. However, on careful inspection, more than $50 \%$ of patients with LE may have mucosal lesions, mainly oral ulcers or nasopharyngeal ulcers ${ }^{24}$. The LE non specific skin manifestations include a wide range of symptoms with different histopathological pictures. The LE non specific skin manifestations are not exclusive to LE disease but are often seen in patients with active SLE but also in several other autoimmune diseases. It is important to screen a patient with CLE for LE non specific symptoms since their presence can imply systemic involvement and progression to $\mathrm{SLE}^{8}$. Patients 
with LE-nonspecific skin manifestations had significantly increased disease activity compared to those with only LEspecific lesions. The number of different skin lesion types also correlated with disease activity. Patients with only one type of lesion had mild disease. An intermediate disease activity was found in the group with two different lesion types. ACLE has a strong association with systemic disease and non-specific skin lesions always indicate disease activity.

The high incidence of CLE emphasizes the importance of following up these patients and recognizing the clinical presentation of disease. Although the cutaneous form of LE has a more indolent course, monitoring the patient's disease is still essential because the disease in some cases progresses to the systemic form, which has a direr prognosis. Early recognition of CLE by the physician translates to early management and, hopefully, to preventing transition of the disease to the systemic form. CCLE and SCLE last for many years and may lead, like SLE, to severe work-related disability and limited life quality. Also, in a small proportion of patients with CLE, SLE develops during the course of their disease, which implies a considerable amount of medical management and costs for the community ${ }^{12}$. Early recognition of patients with CLE who are at risk for SLE development and preventive measures against diseasetriggering factors are important tasks for physicians of patients with CLE. Signs of nephropathy, elevated antinuclear antigen titers, and arthralgia may serve as predictors for transition into SLE. In this study, 61 (28\%) patients had meet $\geq 4$ ACR criteria for SLE. The diagnosis of SLE in these patients does not imply serious systemic disease, since SCLE, ACLE patients commonly meet criteria for SLE based on mucocutaneous findings, immunological markers and serological abnormalities. Both the ACR and SLICC criteria for SLE identify SCLE, ACLE patients with often relatively minimal systemic disease. A 1959 case series by Scott and Rees studying the relationship between SLE and DLE reported that most cases of DLE progressed to SLE within 2 years ${ }^{25}$. Furthermore, early diagnosis often leads to early management, which might also stall the progression of CLE to SLE. Our findings have important implications for physicians and illustrate the importance of follow-up in these patients. While systemic involvement tends to be mild in most patients with CLE, the disease has a major impact on quality of life because the lesions are usually located on the face and the chronic forms can cause irreversible scarring. Moreover, up to $28 \%$ of patients with CCLE are susceptible to developing SLE ${ }^{26}$. The different types of CLE share similar and overlapping pathological features to a greater or lesser extent. There is controversy as to whether SLE and CLE represent different spectrum of the same disease or are distinct disease phenotypes. Cutaneous Lupus Erythematosus (CLE) is an exemplar of a disorder requiring a multidisciplinary approach for its management. It has the potential to intersect with many disciplines and each can contribute to providing the optimum outcome for patients. The disciplines range from the basic sciences through to different organ-specific clinical specialties. CLE has a surprising variety of clinical phenotypes. Therefore, a dermatologist is often the key facilitator for the primary diagnosis with referrals deriving from different disciplines. The subsequent management can take multiple and diverse pathways. Close and coordinated cooperation is important, and an understanding of cutaneous lupus by non-dermatologists is helpful ${ }^{27}$. The Systemic Lupus International Collaborating Clinics (SLICC) criteria1 integrates the cutaneous manifestations of lupus with other the systemic features and is an alternative to the American College of Rheumatology classification ${ }^{28,29}$. SLICC provides a long-needed unified and validated multidisciplinary classification criterion. However, in the dermatology clinic, it is possible to make a diagnosis of CLE in the absence of any features of SLE or with only some but not all of the features needed to define SLE. Furthermore, occasionally, it can be difficult to exactly define into which subcategory of CLE to place a patient when there are overlapping clinical features.

\section{LIMITATIONS}

The study has several limitations. First, it was a hospital-based retrospective study with a relatively smaller sample size. Therefore, a population based study outside the tertiary care setting on the CLE patients will be instructive to validate the findings of the study. Second, making the diagnosis of CLE of some patients involves the clinical judgment of the physician; therefore, there is room for error because of the subjective nature of clinical diagnosis. Milder cutaneous lupus rashes may not be recognized and diagnosed correctly and thus the number presented here may be an underestimate. There are many clinical mimics of cutaneous lupus erythematosus and in absence of histology the diagnosis of CLE may not be certain. Third, there may be undiagnosed cases in the community that have not reached the health care system for screening and diagnosis, and other cases may have received care outside the catchment area. Surveillance outside of the tertiary care setting is imperative for capturing the full spectrum of LE, in order to identify cases.

\section{CONCLUSION}

Photosensitivity was the most common clinical manifestation, whereas ANA was the most frequent autoantibody of the LE patients of this region. Patients with different subtypes of CLE have distinct clinical and pathological characteristics. The onset or concurrence of SLE mandates the involvement of other disciplines depending on organ involvement. In the absence of consensus on a definition that makes it possible to differentiate cutaneous forms of LE from SLE, the dermatologist's role in the correct diagnosis and classification of such patients is fundamental. By recent improvement in diagnostics and therapy options, the prognosis for patients with LE has improved and the impact of this disease on their everyday life has been mitigated. Hopefully, in the future more specific treatments will be available when clinic-pathological correlations have been more clarified.

\section{DISCLOSURE}

All the authors declared no competing interest. 


\section{REFERENCES}

1. Samotij D, Szcz ch J, Kushner CJ, Mowla MR, Da czak-Pazdrowska A, Antiga E, et al. Prevalence of Pruritus in Cutaneous Lupus Erythematosus: Brief Report of a Multicenter, Multinational Cross-Sectional Study. Biomed Res Int. 2018:3491798. doi: $10.1155 / 2018 / 3491798$.

2. Sontheimer RD. The lexicon of cutaneous lupus erythematosus- A review and personal perspective on the nomenclature and classification of the cutaneous manifestations of lupus erythematosus. Lupus. 1997; 6 (2):84-95.

3. Uthman I, Nasr F, Kassak K, and Masri AF. Systemic lupus erythematosus in Lebanon. Lupus. 1999; 8 (9):713-715.

4. Othmani S and Louzir B. Lupus syst' emique chez 24 hommes tunisiens: analyse clinicobiologique et 'evolutive. La Revue de M'edecine Interne. 2002; 23 (12):983-990.

5. Contin-Bordes C, Lazaro E, Pellegrin J, Viallard J, Moreau J, and Blanco P. Lupus 'eryth'emateux syst' emique : de la physiopathologie au traitement. La Revue de M’edecine Interne. 2009; 30 (12):9-13.

6. Cooper GS, Dooley MA, Treadwell EL, St. Clair EW, Parks CG, and Gilkeson GS. Hormonal, environmental, and infectious risk factors for developing systemic lupus erythematosus. Arthritis \& Rheumatology. 1998; 41 (10):1714- 1724.

7. AlSaleh J, Jassim V, ElSayed M, Saleh N, and Harb D. Clinical and immunological manifestations in 151 SLE patients living in Dubai. Lupus. 2008; 17 (1): 62-66.

8. Cardinali C, Caproni M, Bernacchi E, Amato L and Fabbri P. The spectrum of cutaneous manifestations in lupus erythematosus- the Italian experience. Lupus. 2000; 9:417-23.

9. Sontheimer RD, Thomas JR and Gilliam JN. Subacute cutaneous lupus erythematosus: A cutaneous marker for a distinct lupus erythematosus subset. Arch Dermatol. 1979; 115:1409-15.

10. Sontheimer RD. Clinical manifestations of cutaneous lupus erythematosus. In: Wallace DJ, Hahn BH, editors. Dubois' lupus erythematosus. Pennsylvania: Lea \& Febiger. 1993;285-301.

11. Jiménez S, Cervera R, Ingelmo M and Font J. The epidemiology of cutaneous lupus erythematosus. In: Kuhn A, Lehmann P, Ruzicka T, editors. Cutaneous lupus erythematosus. Berlin, Heidelberg, New York: Springer-Verlag. 2004;33-44.

12. Tebbe B and Orfanos CE. Epidemiology and socioeconomic impact of skin disease in lupus erythematosus. Lupus. 1997; 6:96-104.

13. Kuhn A and Ruzicka T. Classification of cutaneous lupus Erythematosus. Cutaneous Lupus Erythematosus. In: Kuhn A, Lehmann P, Ruzicka T, editors. Cutaneous lupus erythematosus. Berlin, Heidelberg, New York: Springer-Verlag. 2005; 53-57.

14. Albrecht J, Taylor L, Berlin JA, Dulay S, Ang G, Fakharzadeh S, et al. The CLASI (Cutaneous Lupus Erythematosus Disease Area and Severity Index): An outcome instrument for cutaneous lupus erythematosus. J Invest Dermatol. 2005; 125:889-894.

15. Kishor N, Boloor R and Sukumar T K. A cross-sectional study of clinico-immunological profile of systemic lupus erythematosus patients in a tertiary care centre in Mangalore. Indian J Allergy Asthma Immunol. 2016; 30 (2):91-94. https://www.ijaai.in/text.asp?2016/30/2/91/195250.

16. Binoy JP, Muhammed F, Kumar N and Razia MV. Clinical profile of systemic lupus erythematosus in North Kerala. J Indian Rheumatol Assoc. 2003; 11:94-97.

17. Cervera R, Khamashta MA, Font J, Sebastiani GD, Gil A, Lavilla P et al. and the European Working Party on Systemic Lupus Erythematosus. Systemic lupus erythematosus: clinical and imunological patterns of disease in a cohort of 1000 patients. Medicine. 1993; 72:113-124.

18. Ginzler EM, Diamond HS, Weiner M, Schlesinger M, Fries JF, Wasner C et al. A multicenter study of outcomes in systemic lupus erythematosus. Entry variables as predictors of prognosis. Arthritis Rheum. 1982; 25:601-611.

19. Jiménez S, Cervera R, Font J and Ingelmo M. The epidemiology of systemic lupus erythematosus. Clin Rev Allergy Immunol. 2003; 25(1):3-12. doi: $10.1385 /$ CRIAI:25:1:3.

20. Lehmann P, Holzle E, Kind P, Goerz G and Plewig G. Experimental reproduction of skin lesions in lupus erythematosus by UVA and UVB radiation. J Am Acad Dermatol. 1990; 22:181-187.

21. Provost TT, Zone JJ and Synowski D. Unusual cutaneous manifestations of systemic lupus erythematosus:Urticaria-like lesions.Correlations with clinical and serological abnormalities. J Invest Dermatol. 1980; 75:495-499.

22. O'Loughlin S, Schoeter AL and Jordan RE. Chronic urticaria-like lesions in systemic lupus erythematosus. Arch Dermatol. 1978; 114:879-883.

23. Weinstein C, Miller MH, Axtens R, Buchanan R, Littlejohn GO. Livedo reticularis associated with increased titres of anticardiolipin antibodies in systemic lupus erythematosus. Arch Dermatol. 1987; 123:596-600.

24. Jonsson R, Heyden G and Westberg NG. Oral mucosal lesions in systemic lupus erythematosus. A clinical, histopathological and immunopathological study. J Rheumatol. 1984; 11:38-42.

25. Scott A and Rees EG. The relationship of systemic lupus erythematosus and discoid lupus erythematosus: A clinical and hematological study. AMA Arch Derm. 1959; 79(4):422-435.

26. Chong BF, Song J, Olsen NJ. Determining risk factors for developing systemic lupus erythematosus in patients with discoid lupus erythematosus. Br J Dermatol. 2012; 166:29-35.

27. Jarrett P and Werth VP. A review of cutaneous lupus erythematosus: Improving outcomes with a multidisciplinary approach. J Multidiscip Healthc. 2019; 12: 419-428. doi: 10.2147/JMDH.S179623.

28. Petri M, Orbai AM, Alarcon GS et al. Derivation and validation of the systemic lupus international collaborating clinics classification criteria for systemic lupus erythematosus. Arthritis Rheum. 2012; 64 (8):2677-2686. doi:10.1002/art.34473

29. Tan EM, Cohen AS, Fries JF et al. The 1982 revised criteria for the classification of systemic lupus erythematosus. Arthritis Rheum. 1982; 25(11):1271-1277. 